\title{
Direct Observation of the Bandwidth Control Mott Transition in the $\mathrm{NiS}_{2-x} \mathrm{Se}_{x}$ Multiband System
}

\author{
H. C. Xu, ${ }^{1}$ Y. Zhang, ${ }^{1}$ M. Xu, ${ }^{1}$ R. Peng, ${ }^{1}$ X. P. Shen, ${ }^{1}$ V. N. Strocov, ${ }^{2}$ M. Shi, ${ }^{2}$ M. Kobayashi, ${ }^{2}$ \\ T. Schmitt, ${ }^{2}$ B. P. Xie, ${ }^{1, *}$ and D. L. Feng ${ }^{1, \dagger}$ \\ ${ }^{1}$ State Key Laboratory of Surface Physics, Department of Physics, and Advanced Materials Laboratory, \\ Fudan University, Shanghai 200433, People's Republic of China \\ ${ }^{2}$ Swiss Light Source, Paul Scherrer Institute, CH-5232 Villigen PSI, Switzerland
}

(Received 9 July 2013; published 25 February 2014)

\begin{abstract}
The bulk electronic structure of $\mathrm{NiS}_{2-x} \mathrm{Se}_{x}$ has been studied across the bandwidth-control Mott transition (BCMT) using soft $\mathrm{x}$-ray angle-resolved photoemission spectroscopy. We show that Se doping does not alter the Fermi surface volume. When approaching the insulating phase with decreasing Se concentration, we observed that the Fermi velocity continuously decreases. Meanwhile, the weight of the coherent quasiparticle, which sits on a large incoherent spectrum, continuously decreases and is transferred to higher binding energies, until it suddenly disappears across the Mott transition. In the insulating phase, there is still finite spectral weight at the Fermi energy, but it is incoherent and dispersionless due to strong correlations. Our results provide a direct observation of the distinct characters of BCMT in a multiband non-half-filled system.
\end{abstract}

DOI: 10.1103/PhysRevLett.112.087603

PACS numbers: 79.60.-i, 71.20.-b, 71.30.+h

The Mott transition is a metal-insulator transition (MIT) due to the strong electron correlations. From a band picture, Brinkman and Rice proposed that the Mott transition occurs when the quasiparticle mass diverges and its weight diminishes [1]. While in the single-band Hubbard model, it occurs when the on-site Coulomb repulsion $U$ is large enough (compared with the bandwidth $W$ ), and splits a half-filled band into a filled lower Hubbard band and an empty upper Hubbard band [2]. These two pictures are unified in the dynamical mean-field theory (DMFT) calculations for intermediate $U$ [3], where the metallic phase exhibits a complex spectral function with Hubbard bands at high energies and quasiparticle bands at low energies. The Mott transition occurs when the quasiparticle mass diverges and its spectral weight is transferred completely to the Hubbard bands with increasing $U$ or decreasing $W$.

One could realize the Mott transition by controlling either the filling or the bandwidth of the Hubbard bands [2]. In filling control, a partially filled Hubbard band is created by doping, as in the cuprate superconductors. In bandwidth control, $W$ is tuned against $U$ by applying physical or chemical pressure, as inferred indirectly from the transport properties of $\mathrm{V}_{2} \mathrm{O}_{3}, \mathrm{NiS}_{2-x} \mathrm{Se}_{x}$, etc. [4,5]. The spectral weight transfer and band evolution predicted by DMFT have been observed in $\mathrm{Ca}_{1-x} \mathrm{Sr}_{x} \mathrm{VO}_{3}$ [6,7]; however, since it is metallic over the entire doping range, MIT is never reached. So far, there lacks direct observation of the momentum-resolved band structure evolution across the bandwidth-control Mott transition (BCMT).

Real materials are seldom of single band, whose Mott transition requires more sophisticated multiband calculations $[8,9]$. Because of its complexity, a comprehensive theoretical understanding of the Mott transition in multiband systems is yet to be achieved and examined by the experiments. $\mathrm{NiS}_{2-x} \mathrm{Se}_{x}$ is a prototypical non-half-filled multiband BCMT system, with the MIT at $x_{c} \sim 0.4$ [10-12] [Fig. 2(a)]. According to the previous transport measurements, the approach to the antiferromagnetic insulator (AFI) phase with doping is characterized by an effective mass enhancement in the paramagnetic metal (PM) regime [Fig. 2(a)], and a decrease in carrier density in the antiferromagnetic metal (AFM) regime [13]. The decrease in carrier density was attributed to the shrinkage of the Fermi surface (FS), but the FS has never been resolved. On the other hand, in previous ultraviolet angleresolved photoemission (UV-ARPES) studies, a narrow band of a many-body nature develops at the Fermi energy $\left(E_{F}\right)$ in metallic phase [14]. However, it persists in the insulating regime, which was attributed to possible carrier doping by Se/S vacancies [15], or some surface states, since UV-ARPES is a surface sensitive technique [16]. Recently, another scenario was proposed against the BCMT [17], where the gap was induced by the bonding-antibonding splitting of S-S (Se-Se) dimer bands. The electronic structure evolution of this prototype system across its MIT thus needs a thorough examination.

We have investigated the bulk electronic structure of $\mathrm{NiS}_{2-x} \mathrm{Se}_{x}$ by soft x-ray angle-resolved photoemission spectroscopy (SX-ARPES). The SX-ARPES provides much better $k_{z}$ resolution and bulk sensitivity as its main advantages over UV-ARPES [18-20]; it is thus suitable for studying the bulk electronic structure of $\mathrm{NiS}_{2-x} \mathrm{Se}_{x}$ with a cubic structure. The FSs are resolved, which show that the Luttinger volume in the metallic regime is doping 
independent. When approaching the AFI phase, we observed that the bandwidths and the Fermi velocities $\left(V_{F}\right)$ of the coherent quasiparticle bands decrease and eventually diminish across the MIT. Meanwhile, the coherent spectral weight is transferred into higher binding energies, and eventually depleted entirely at the MIT. In contrast, there is always large incoherent spectral weight over the studied doping range. Our data reveal the distinct electronic signature of BCMT in a non-half-filled multiband system.

Single crystals of $\mathrm{NiS}_{2-x} \mathrm{Se}_{x}$ were synthesized with dopings covering all phases in the phase diagram [Fig. 2(a)] [21]. Those with $x>0.71$ were grown by the chemical vapor transport method [22], and the others by the Te flux method [23]. The actual dopings were determined by energy dispersive $\mathrm{x}$-ray spectroscopy and confirmed by resistivity measurements [24], consistent with Ref. [21]. The SX-ARPES data were taken with $p$-polarized light at the Advanced Resonant Spectroscopies (ADRESS) beam
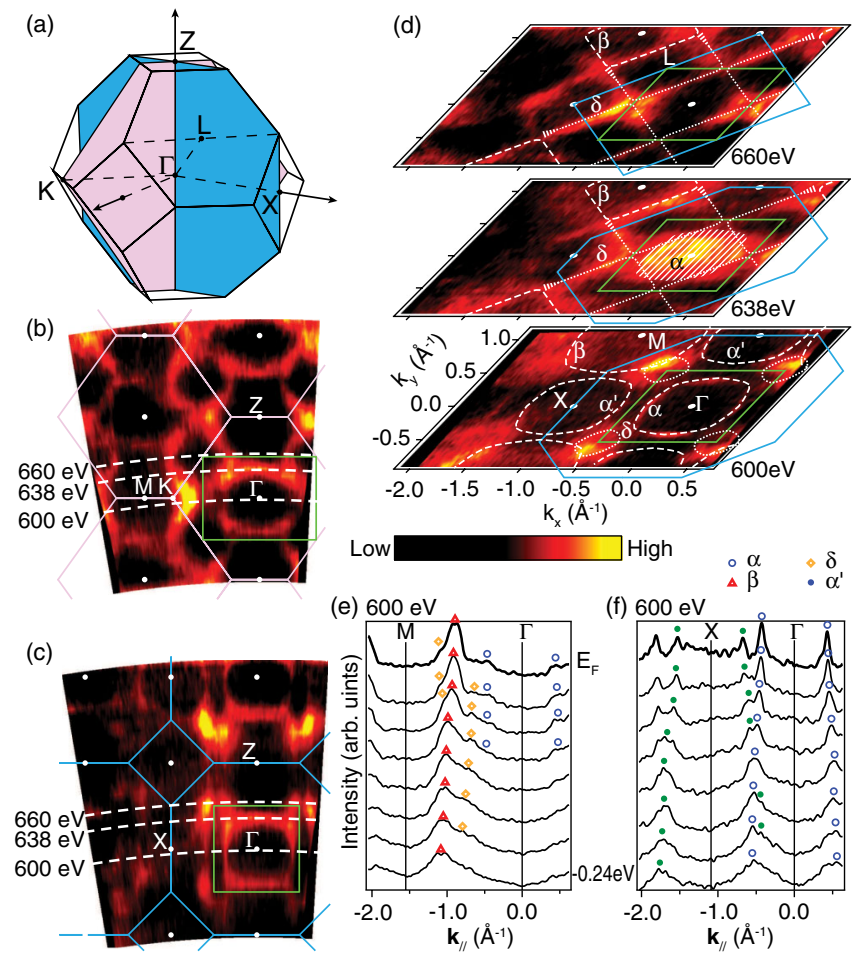

FIG. 1 (color online). Electronic structure of $\mathrm{NiS}_{2-x} \mathrm{Se}_{x}$ $(x=1.67)$. (a) The Brillouin zone (BZ) for the face-centeredcubic sublattice of $\mathrm{Ni}^{2+}$. (b), (c) The FS mapping in the $\Gamma-M-Z$ plane, and the $\Gamma-X-Z$ plane, respectively. The green rectangles illustrate the 1st BZ for the simple cubic lattice. (d) FS slices in the $\Gamma-X-M$ plane $(h \nu=600 \mathrm{eV})$, the plane at the half $\mathrm{BZ}$ height $(660 \mathrm{eV})$, and a intermediate plane $(638 \mathrm{eV})$, respectively, as indicated by dashed curves in (b) and (c). All these intensities were integrated over $E_{F} \pm 15 \mathrm{meV}$. The FS sheets are shown by dashed curves. Part of the $\delta$ FS is hard to trace, as marked by the thicker dashed curves. The hatched area indicates the Fermi patch formed by cutting along the FS. (e),(f) Momentum distribution curves near $E_{F}$ along $\Gamma-M$, and $\Gamma-X$, respectively. line of the Swiss Light Source (SLS) [25]. The overall energy resolution is $\sim 80 \mathrm{meV}$. Samples were cleaved in situ at the (001) plane and measured at $11 \mathrm{~K}$ under a vacuum better than $5 \times 10^{-11}$ mbar. Details of the characterization of the samples from the same batch are provided in the Supplemental Material [24].

The Brillouin zone (BZ) corresponding to the facecentered-cubic (fcc) sublattice of $\mathrm{Ni}^{2+}$ in $\mathrm{NiS}_{2-x} \mathrm{Se}_{x}$ is shown in Fig. 1(a) [24]. Along the two high symmetric $\Gamma-M-Z$ (pink) and $\Gamma-X-Z$ (blue) planes [Fig. 1(a)], $k_{z}$ dependent photoemission intensity distributions of $\mathrm{NiS}_{0.33}$ $\mathrm{Se}_{1.67}$ at $E_{F}$ are shown in Figs. 1(b) and 1(c), with the finite photon momentum corrected. After assuming the inner potential $\left(V_{0}\right)$ of $25 \mathrm{eV}$, the periodicity of the $\mathrm{FS}$ matches that of the BZ for the fcc sublattice, rather than that of the BZ for simple cubic lattice [24]. Three cross sections of the FS taken with different photon energies in Fig 1(d) show their three-dimensional structure. The dispersions near $E_{F}$ are illustrated by the momentum distribution curves (MDCs) along $\Gamma-M$ and $\Gamma-X$ in Figs. 1(e) and 1(f), respectively. We find that the FSs in the $\Gamma-X-M$ plane [lower panel of Fig. 1(d)] are composed of three sets: (i) a hole pocket centered at $\Gamma$ contributed by the band $\alpha$, and a hole pocket centered at $X$ noted as $\alpha^{\prime}$ that is the counterpart of $\alpha$ in the 2nd simple cubic BZ; (ii) an electron pocket contributed by the band $\beta$ centered at $M$; (iii) four small and weak electron pockets at the corner of the simple cubic BZ,
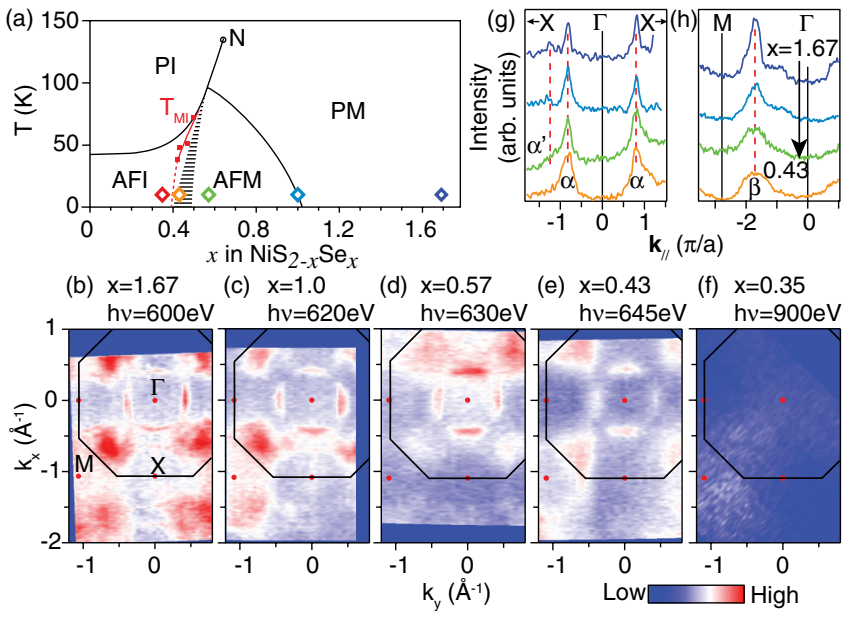

FIG. 2 (color online). (a) The phase diagram of $\mathrm{NiS}_{2-x} \mathrm{Se}_{x}$ reproduced from Ref. [21], on which the MIT temperatures $\left(T_{M I}\right)$ of our samples (red squares) are plotted as a function of doping [24]. The averaged dopings for the sample batches are marked by the diamonds. AFI, AFM, PI, and PM stand for antiferromagnetic insulator, antiferromagnetic metal, paramagnetic insulator, and paramagnetic metal, respectively. (b)-(f) The FS mapping for various dopings in the $\Gamma-X-M$ plane. The intensities are integrated over $E_{F} \pm 15 \mathrm{meV}$ and then normalized by the integrated intensity at $-4 \mathrm{eV}$. (g),(h) The MDC's at $E_{F}$ normalized by spectrum intensities for various dopings along $\Gamma-X$, and $\Gamma-M$, respectively. 

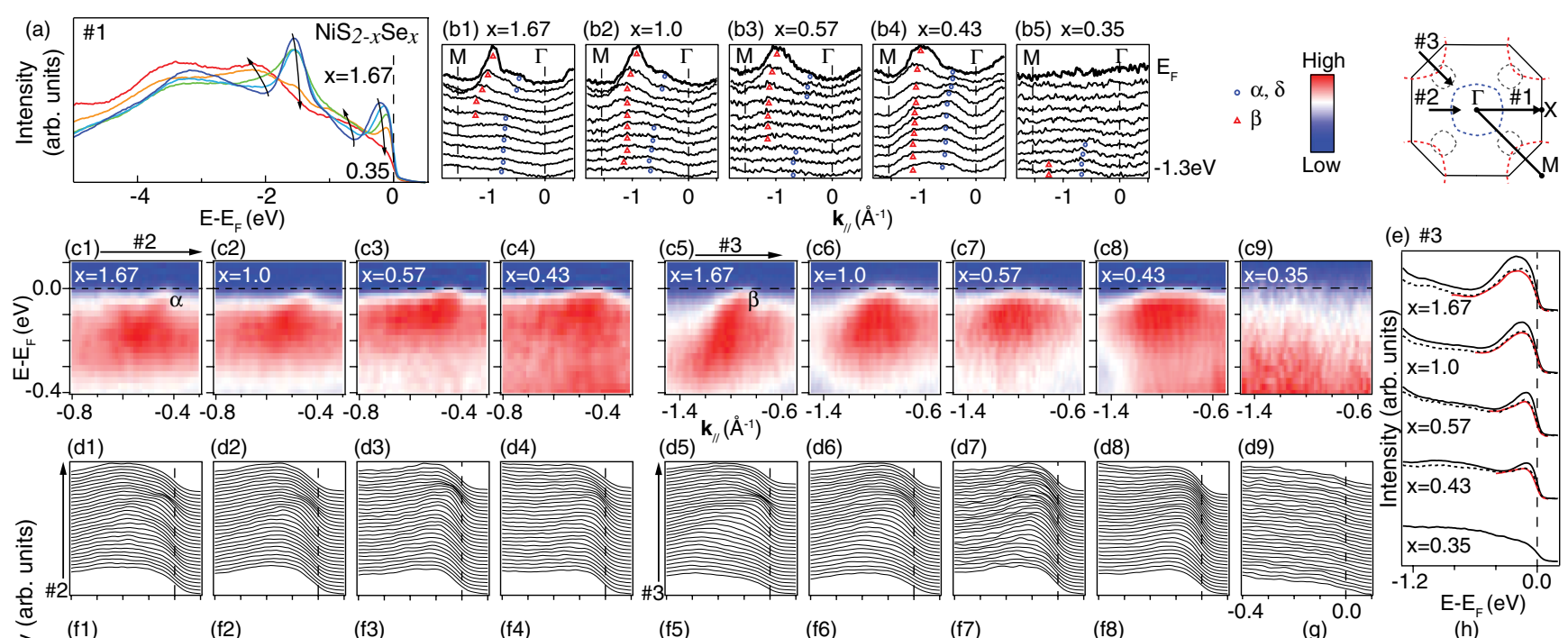

$-1 \quad 0$ $\mathbf{k}_{/ /}\left(\AA^{-1}\right)$ $-1$

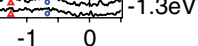
Low $M$
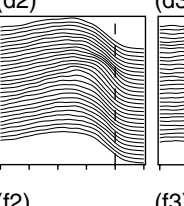

(d4)
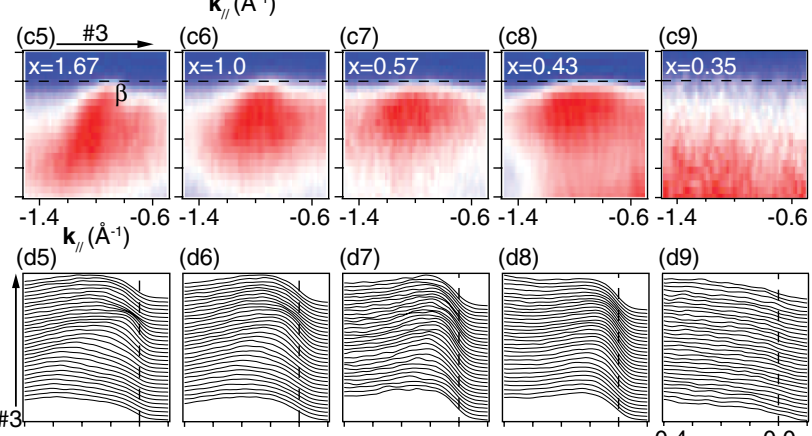

(e) \#3

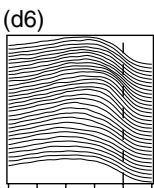

(d7)

\begin{abstract}
(d8)
\end{abstract}
$(\mathrm{d} 9)$

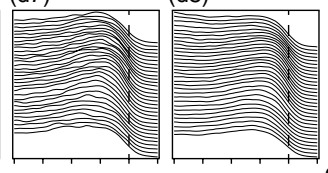

(d9)
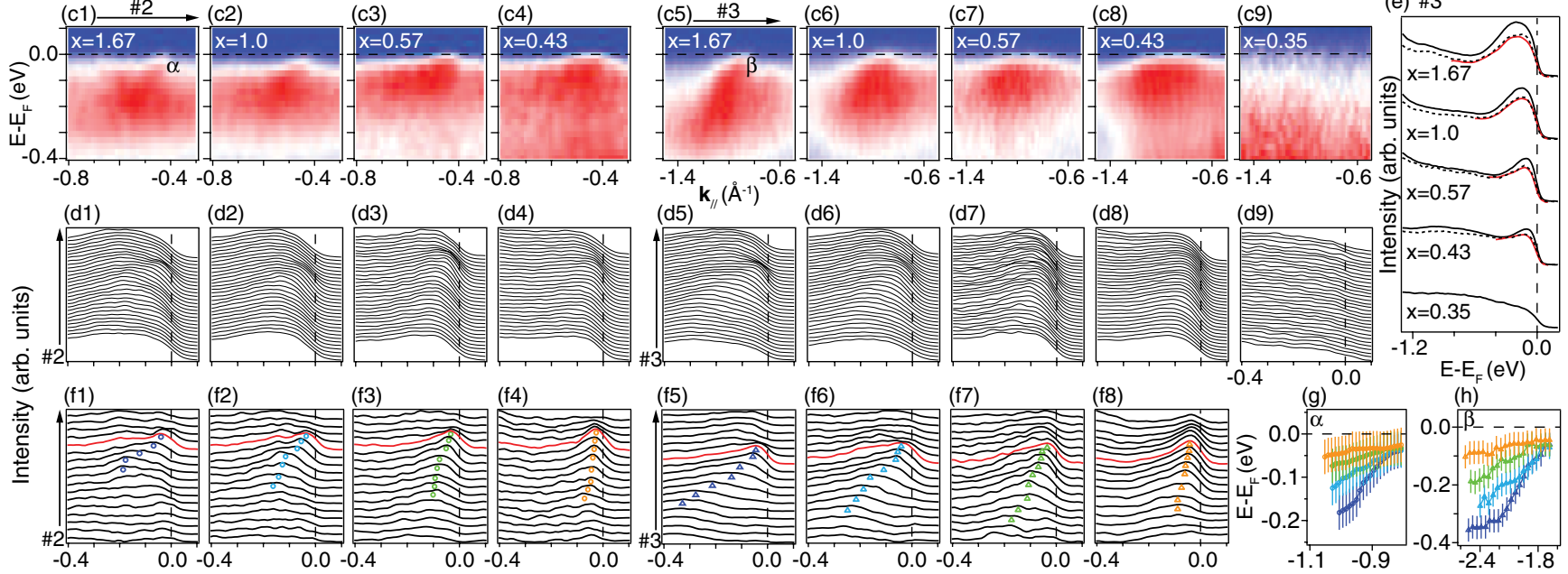

(f4) (f5)

(f6)
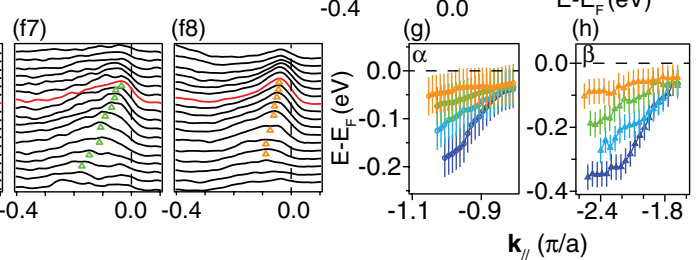

FIG. 3 (color online). (a) Doping dependence of the photoemission spectra integrated along $M-\Gamma-X$ (cut \#1 in the inset) normalized by total intensity. (b1)-(b5) MDC's along $\Gamma-M$ for doping $x=1.67,1.0,0.57,0.43$, and 0.35 , respectively. The blue open circle and red triangle marks stand for features corresponding to the bands $\alpha$ or $\delta$, and $\beta$, respectively. (c1)-(c4) Intensity along cut \#2 as indicated in the inset for doping $x=1.67,1.0,0.57$, and 0.43, respectively. (c5)-(c9) Intensity along cut \#3 for doping $x=1.67,1.0,0.57,0.43$, and 0.35 , respectively. (d1)-(d9) Energy distribution curves (EDC's) corresponding to (c1)-(c9). (e) Integrated EDC's along cut \#3 (black solid curves) plotted with EDCs at $\Gamma$ (black dashed curves), and the incoherent and dispersionless background (red solid curves). (f1)-(f8) The EDCs of (c1)-(c8) after subtracting the incoherent background in panel (e). The open circles and triangles trace the dispersions of $\alpha$ and $\beta$, respectively. (g),(h) The extracted dispersions, as color coded in (f1)-(f8), for bands $\alpha$ and $\beta$, respectively. The inset illustrates the cuts \#1, \#2, and \#3 in the $\Gamma-X-M$ plane.

contributed by the band $\delta$. These detailed electronic structures are not captured by the recent local-density approximation (LDA) calculations, which give just one electron pocket around $\Gamma$ in $\mathrm{NiSe}_{2}$ [17]. Hereafter, we focus on the data in the $\Gamma-X-M$ plane, which represent the general electronic structure.

Noting the lattice constant varies with doping from $5.96\left(\mathrm{NiSe}_{2}\right)$ to $5.69 \AA\left(\mathrm{NiS}_{2}\right)$ [26], we pinpoint the photon energy that corresponds to the $\Gamma-X-M$ plane for each doping from high- $k_{z}$-resolution photoemission intensity distributions. The FS mapping in the $\Gamma-X-M$ plane for all measured dopings is shown in Figs. 2(b)-2(f), and there is no detectable variation of FS in the metallic regime, except for the fading of the $\alpha^{\prime}$ pocket possibly due to the enhanced dimer disorder. The Fermi crossings along $\Gamma-X$ [Fig. 2(g)] and $\Gamma-M$ [Fig. 2(h)] appear invariant. On the other hand, the intensity at $E_{F}$ decreases gradually with decreasing $x$, and the FSs disappear in the insulating phase. The doping independent FS volume proves the isovalent nature of the S/Se substitution, which also suggests the validity of the Luttinger theorem with progressively increasing correlations. Therefore, the decrease in carrier density is not due to the proposed Luttinger volume reduction [13,27], but the diminishing spectral weight at $E_{F}$.

In Fig. 3(a), the valence band spectra integrated over cut \#1 show two narrow peaks at $-1.6 \mathrm{eV}$ and just below $E_{F}$, respectively. These peaks are composed of both Ni $3 d$ and $\mathrm{S} / \mathrm{Se} p$ states according to our resonant photoemission data, confirming the charge transfer nature of the band structure $[11,24,28,29]$. With decreasing $x$, one could observe a reduction of the peak near $E_{F}$, accompanied by a relative enhancement of spectral weight at $0.5-1 \mathrm{eV}$ below $E_{F}$. The peak at $-1.6 \mathrm{eV}$ behaves almost identically; its spectral weight seems to be transferred to higher binding energies as well, which indicates that all the $3 d$ electrons are correlated [15]. The peak near $E_{F}$ moves towards $E_{F}$ with decreasing $x$, as observed by scanning tunneling spectroscopy before [27]. It disappears in our SX-ARPES data for $x=0.35$, the AFI phase. The narrow peak near $E_{F}$ observed previously by UV-ARPES in the AFI phase may be originated from the surface $[14,15]$.

In the common picture of BCMT, bandwidth is the key parameter whose evolution can only be extracted from the bare band dispersion. Nevertheless, as illustrated before both theoretically [30,31] and experimentally for the blue 
bronze and cuprate [32-34], the bare band dispersion is manifested as the MDCs centroid of the broad incoherent spectrum. Indeed, the MDCs in Figs. 3(b1)-3(b5) show highly dispersive features with rather weak doping dependence for $\mathrm{NiS}_{2-x} \mathrm{Se}_{x}$; thus, presumably the bare bandwidth is not strongly influenced by the Se substitution. Such a general feature is captured in the recent band calculations, where the overall bandwidth decreases by less than $7 \%$ from $\mathrm{NiSe}_{2}$ to $\mathrm{NiS}_{2}$ [17]. On the other hand, in the first $0.4 \mathrm{eV}$ below $E_{F}$, one could observe the doping dependence of the spectral intensity distribution along cut \#2 [Figs. 3(c1)-3(c4)] and \#3 [Figs. 3(c5)-3(c9)]. The corresponding energy distribution curves are shown in Figs. 3(d1)-3(d9), respectively, where the spectra of the metallic samples are made of a large incoherent and dispersionless broad background and a weak dispersive feature, while that of the insulating sample is featureless.

To obtain the incoherent and dispersionless background, one could either take the broad spectrum at $\Gamma$ (since one would not expect any feature near $E_{F}$ ), or combine the momentum-independent part at individual binding energies [24]. The similar results from these two methods are plotted in Fig. 3(e), together with the angle-integrated spectra along cut \#3 for comparison. After subtracting the background, one can readily identify that the $V_{F}$ of the $\alpha$ and $\beta$ bands decrease with decreasing doping in Figs. 3(f1)-3(f8), as summarized in Figs. 3(g)-3(h). We note that the dispersionless feature shifts towards $E_{F}$ with decreasing $x$ [Fig. 3(e)], following the renormalization of the coherent bands, which implies that the incoherent weight could be some shake-off side bands from the coherent quasiparticles by strong correlations.

The $V_{F}$ of the quasiparticle dispersions along both the cuts \#2 and \#3 are summarized in Fig. 4(a). They drop rapidly when approaching the Mott transition, and most likely diminish at the transition based on its trend. Such a strong enhancement or even diverging quasiparticle mass near $x_{c}$ is consistent with previous transport measurements [13]. Therefore, even though the bare band cannot be extracted accurately, the observed $V_{F}$ or quasiparticle bandwidth renormalization provides a straightforward illustration of BCMT [2]. Particularly, the rate of band renormalization is much faster than the bare bandwidth variation, highlighting the dominant role of the increased strong correlations.

In Fig. 4(b), we plot the density of state represented by the integrated spectral weight at $E_{F}$ along the cut \#1 in Fig. 3. It shows similar doping dependence as the specific heat linear coefficient $\gamma$ reproduced from Ref. [13]. Both quantities show a peak at around $x=1$, which is the critical point between the PM and AFM phases, where the spin fluctuations lead to a large $\gamma$ value [13]. The density of state at $E_{F}$ does not exhibit any anomaly across the Mott transition, and it is still finite even for the insulator. Similarly in Fig. 4(c), the incoherent spectral weight

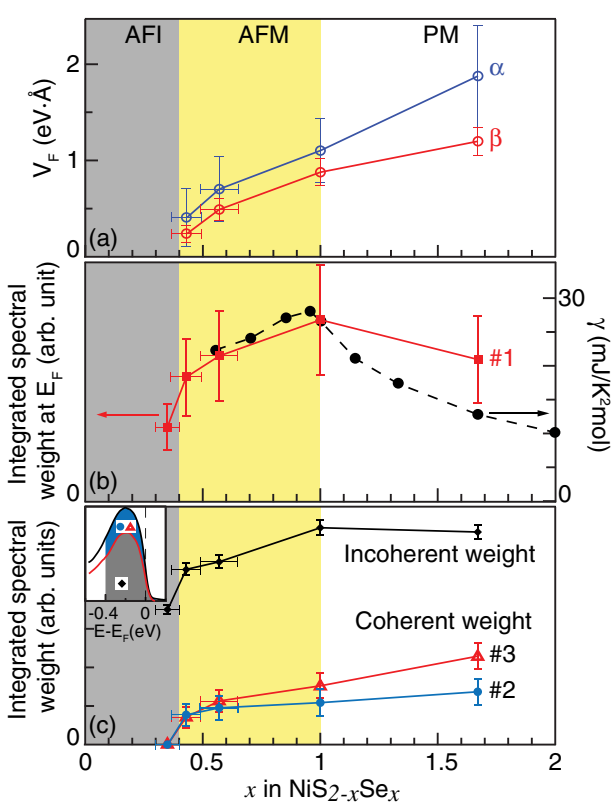

FIG. 4 (color online). (a) The doping dependence of $V_{F}$ of bands $\alpha$ and $\beta$ [Figs. 3(f1)-3(f8)]. (b) The doping dependence of spectral weight at $E_{F}$ integrated over $E_{F} \pm 40 \mathrm{meV}$ along the path \#1 in Fig. 3, which resembles the specific heat linear coefficient $\gamma$ [13]. (c) The doping dependence of the incoherent and dispersionless background (gray area in the inset) and the dispersive spectral weight along cuts \#2 and \#3 (blue area in the inset) over the energy range $\left[E_{F}-0.4 \mathrm{eV}, E_{F}+0.1 \mathrm{eV}\right]$.

dominates the spectrum at lower dopings, and crosses the Mott transition smoothly. In contrast, the coherent weight diminishes at the Mott transition for both the cuts \#2 and \#3. Intriguingly, the decreasing rates are accelerated near the Mott transition for both the coherent and incoherent weight.

The spectral weight transfer and band narrowing characters of the BCMT in $\mathrm{NiS}_{2-x} \mathrm{Se}_{x}$ partially resemble the physical picture established in a single band Hubbard model, and the picture of Brinkman and Rice. However, the anomalously large incoherent spectral weight found here (observed in optical conductivity as well [35]) is beyond these models based on single band. Such an absence of a clean gap is likely related to the non-halffilled multiband nature of $\mathrm{NiS}_{2-x} \mathrm{Se}_{x}$. It requires more sophisticated multiband calculations with proper consideration of the strong correlations. In fact, the BCMT of this system could alternatively be viewed as a coherentincoherent transition, where the incoherent spectral weight gradually dominates at $E_{F}$ due to rapidly increasing strong correlations. In this way, the system is converted from a bad metal to a bad insulator, as found by resistivity and optical measurements [21,36]. Similar coherent-incoherent MIT characterized by finite incoherent spectral weight at $E_{F}$ in the high temperature insulating phase has been observed before in polaronic systems [37-39]. It would be instructive to investigate the MIT from the AFM phase to the AFI/PI 
phase in $\mathrm{NiS}_{2-x} \mathrm{Se}_{x}$ with increasing temperature in future SX-ARPES experiments.

To summarize, we have presented the detailed electronic structure evolution across the BCMT in $\mathrm{NiS}_{2-x} \mathrm{Se}_{x}$, a multiband non-half-filled system. We found that the FS volume is unaffected by sulfur doping in the PM and AFM phases, and the bare bandwidth is just moderately narrowed. The increased correlations transfer the spectral weight into higher binding energies, reduce the coherent bandwidth, suppress the coherent spectral weight, and eventually lead to the Mott transition that is characterized by a divergent effective quasiparticle mass and a depleted coherent weight at $E_{F}$. Moreover, the insulating phase is characterized by the finite incoherent spectral weight at $E_{F}$ without opening a charge gap. These results deepen our understanding of Mott transitions.

We gratefully acknowledge Professor Hide Takagi for providing some single crystals to us years ago at the beginning of this project, and Professor Zhengyu Weng, Professor Qianghua Wang, and Professor David Singh for helpful discussions. This work is supported in part by the National Science Foundation of China and National Basic Research Program of China (973 Program) under the Grant No. 2012CB921400, No. 2011CB921802, and No. 2011 CBA00112.

*bpxie@fudan.edu.cn †dlfeng@fudan.edu.cn

[1] W. F. Brinkman and T. M. Rice, Phys. Rev. B 2, 4302 (1970).

[2] M. Imada, A. Fujimori, and Y. Tokura, Rev. Mod. Phys. 70, 1039 (1998).

[3] X. Y. Zhang, M. J. Rozenberg, and G. Kotliar, Phys. Rev. Lett. 70, 1666 (1993).

[4] W. Bao, C. Broholm, S. A. Carter, T. F. Rosenbaum, G. Aeppli, S. F. Trevino, P. Metcalf, J. M. Honig, and J. Spalek, Phys. Rev. Lett. 71, 766 (1993).

[5] J. M. Honig and J. Spalek, Chem. Mater. 10, 2910 (1998).

[6] I. H. Inoue, I. Hase, Y. Aiura, A. Fujimori, Y. Haruyama, T. Maruyama, and Y. Nishihara, Phys. Rev. Lett. 74, 2539 (1995).

[7] T. Yoshida, M. Hashimoto, T. Takizawa, A. Fujimori, M. Kubota, K. Ono, and H. Eisaki, Phys. Rev. B 82, 085119 (2010).

[8] P. Werner, E. Gull, M. Troyer, and A. J. Millis, Phys. Rev. Lett. 101, 166405 (2008).

[9] L. de'Medici, J. Mravlje, and A. Georges, Phys. Rev. Lett. 107, 256401 (2011)

[10] J. A. Wilson and G. D. Pitt, Philos. Mag. 23, 1297 (1971).

[11] S. R. Krishnakumar and D. D. Sarma, Phys. Rev. B 68, 155110 (2003).

[12] M. Matsuura, H. Hiraka, K. Yamada, and Y. Endoh, J. Phys. Soc. Jpn. 69, 1503 (2000).
[13] S. Miyasaka, H. Takagi, Y. Sekine, H. Takahashi, N. Mori, and R. J. Cava, J. Phys. Soc. Jpn. 69, 3166 (2000).

[14] A. Y. Matsuura, Z. X. Shen, D. S. Dessau, C. H. Park, T. Thio, J. W. Bennett, and O. Jepsen, Phys. Rev. B 53, R7584 (1996).

[15] A. Y. Matsuura, H. Watanabe, C. Kim, S. Doniach, Z.-X. Shen, T. Thio, and J. W. Bennett, Phys. Rev. B 58, 3690 (1998).

[16] D. D. Sarma et al., Phys. Rev. B 67, 155112 (2003).

[17] J. Kuneš, L. Baldassarre, B. Schächner, K. Rabia, C. A. Kuntscher, Dm. M. Korotin, V. I. Anisimov, J. A. McLeod, E. Z. Kurmaev, and A. Moewes, Phys. Rev. B, 81, 035122 (2010).

[18] V. N. Strocov, J. Electron Spectrosc. Relat. Phenom. 130, 65 (2003).

[19] V. N. Strocov, M. Shi, M. Kobayashi, C. Monney, X. Wang, J. Krempasky, T. Schmitt, L. Patthey, H. Berger, and P. Blaha, Phys. Rev. Lett. 109, 086401 (2012).

[20] E. Razzoli et al., Phys. Rev. Lett. 108, 257005 (2012).

[21] X. Yao, J. M. Honig, T. Hogan, C. Kannewurf, and J. Spałek, Phys. Rev. B 54, 17469 (1996).

[22] R. J. Bouchard, J. L. Gillson, and H. S. Jarrett, Mater. Res. Bull. 8, 489 (1973).

[23] X. Yao and J. M. Honig, Mater. Res. Bull. 29, 709 (1994).

[24] See Supplemental Material at http://link.aps.org/ supplemental/10.1103/PhysRevLett.112.087603 for information on the growth and characterization of $\mathrm{NiS}_{2-x} \mathrm{Se}_{x}$ single crystals, the crystal structure and the low energy electron diffraction pattern, the resonant photoemission study, and details on determining the incoherent and dispersionless background.

[25] V. N. Strocov et al., J. Synchrotron Radiat. 17, 631 (2010).

[26] F. Gautier, G. Krill, M. F. Lapierre, P. Panissod, and C. Robert, Phys. Lett. A 5331 (1975).

[27] K. Iwaya, Y. Kohsaka, S. Satow, T. Hanaguri, S. Miyasaka, and H. Takagi, Phys. Rev. B 70, 161103 (2004).

[28] C. Guillot, Y. Ballu, J. Paigné, J. Lecante, K. P. Jain, P. Thiry, R. Pinchaux, Y. Pétroff, and L. M. Falicov, Phys. Rev. Lett. 39, 1632 (1977).

[29] A. Fujimori, K. Mamiya, T. Mizokawa, T. Miyadai, T. Sekiguchi, H. Takahashi, N. Môri, and S. Suga, Phys. Rev. B 54,16329 (1996).

[30] A. S. Mishchenko and N. Nagaosa, Phys. Rev. Lett. 93, 036402 (2004).

[31] O. Rösch and O. Gunnarsson, Eur. Phys. J. B 43, 11 (2005).

[32] M. Grioni, L. Perfetti, and H. Berger, J. Electron Spectrosc. Relat. Phenom. 137-140, 417 (2004).

[33] K. Yang et al., Phys. Rev. B 73, 144507 (2006).

[34] B. P. Xie et al., Phys. Rev. Lett. 98, 147001 (2007).

[35] A. Perucchi et al., Phys. Rev. B 80, 073101 (2009).

[36] R. L. Kautz, M. S. Dresselhaus, D. Adler, and A. Linz, Phys. Rev. B, 6, 2078 (1972).

[37] P. Kwizera, M. S. Dresselhaus, and D. Adler, Phys. Rev. B, 21, 2328 (1980).

[38] T. Valla et al., Nature (London) 417, 627 (2002).

[39] Y. Zhang et al., Phys. Rev. B 82, 165113 (2010). 